\title{
Amaldi Meeting Introduction
}

\author{
Barry C. Barish \\ California Institute of Technology \\ Pasadena, CA 91125
}

Welcome to Caltech and the $3^{\text {rd }}$ Edoardo Amaldi Conference on Gravitational Waves. Obviously, something must be very interesting to bring more than 250 scientists from around the world to Pasadena in July for this particular meeting. In fact, July in Southern California does have many attractions, in addition to the good weather and cool nights. For this conference, we have arranged a visit to the new Getty Museum on our excursion day. This is meant to make your stay more pleasant, but is not the real reason we have gathered here. This meeting addresses the detection of gravitational waves, a much-anticipated event.

Einstein predicted Gravitational Waves in 1916, as a result of his theory of gravity. This new description, involving curved space-time, solved the fundamental problem in Newtonian gravity of instantaneous action at a distance. Other effects of general relativity have been observed, including the recent advances in gravitational lensing that are becoming so important in astrophysics. Gravitational waves, themselves, have been indirectly observed in the beautiful experiment of Hulse and Taylor, by measuring the gradual speed up of the period of the binary neutron star system PS 1913+16, and showing that it precisely agrees with the effect predicted from the radiation of gravitational waves.

The experimental challenge of detecting gravitational waves has been with us since Einstein introduced the concept. Unfortunately, the effects due to gravitational waves are so small that a self-contained laboratory experiment appears unfeasible. Instead, the present attempts are to detect gravitational waves resulting from some astrophysical or cosmological effect. Possible sources include supernovae, binary inspiral of compact objects like neutron stars or black holes, spinning neutron stars, or even gravitational waves that were emitted in the first instants of the early Universe.

Sensitive techniques have been developed over the past three decades, first using resonant bars and more recently, suspended mass interferometers. Vigorous research has been done to develop these techniques and large ambitious experiments are now being implemented. With the world-wide effort and the very large interferometers being developed the community has grown and there is a growing need for this

CP523, Gravitational Waves: Third Edoardo Amaldi Conference, edited by S. Meshkov (C) 2000 American Institute of Physics 1-56396-944-0/00/\$17.00 
emerging field to organize itself for collaboration, exchange of information and to represent the field.

For these reasons, the gravitational wave community organized itself a couple years ago forming the Gravitational Wave International Committee (GWIC, for short) in order to help foster the international aspects of this emerging field. One of the first tasks was to create a conference specifically for this field. Rather than create a new conference, GWIC decided to 'evolve' a successful existing topical workshop, the Amaldi meeting that had been held twice, once at Frascati, Italy and then at CERN in Switzerland.

GWIC has become an important committee for the field of gravitational waves. It has representatives from all major projects in the world - resonant bars, interferometers and also the space based initiative. The committee is developing a formal tie with IUPAP (the International Union of Physics and Applied Physics), which is the highest body of scientists representing international community. The relationship is through an Associated Commission - AC2 that covers general relativity; as well as being linked to PaNAGIC, a newly formed committee in IUPAP to represent particle astrophysics and related fields. We expect to seek IUPAP sponsorship of the next Amaldi meeting, which will be in Perth, Australia in 2001.

This Amaldi meeting at Caltech represents the first in the series under the auspices of GWIC and has been organized as a true international conference. To accomplish that, we have followed IUPAP guidelines for international conferences in terms of distribution of delegates, speakers, etc.

The program of the meeting covers astrophysics sources, resonant bar detectors (coincidences), suspended mass interferometers (new generation becoming operational over the next few years), and space based experiments (LISA) (which hopefully will become a reality over the coming decade). It covers technical details, as well as data analysis approaches and techniques. Syd Meshkov has put the conference together very capably with advice from an international organizing committee.

Lastly, I would like to comment that GWIC has kept the title Eduardo Amaldi Conference in tribute to the fact that Eduardo Amaldi was such an important figure in $20^{\text {th }}$ century experimental physics, and that he spent the later part of his career pioneering this new field and developing the techniques to search for gravitational waves. We are fortunate today to have his son Ugo Amaldi, an old friend, here to talk and give us a tribute to his father.

Hopefully, this will be the first in a long series of successful Amaldi meetings sponsored by GWIC that will trace the progress in this exciting field as gravitational waves are directly detected and become a new tool for studying fundamental questions in physics and astronomy. 